\title{
The 2-Pebbling Property of the Middle Graph of Fan Graphs
}

\author{
Yongsheng Ye, Fang Liu, and Caixia Shi \\ School of Mathematical Sciences, Huaibei Normal University, Huaibei, Anhui 235000, China \\ Correspondence should be addressed to Yongsheng Ye; yeysh66@163.com
}

Received 29 April 2014; Accepted 7 July 2014; Published 22 July 2014

Academic Editor: Ying Tan

Copyright (C) 2014 Yongsheng Ye et al. This is an open access article distributed under the Creative Commons Attribution License, which permits unrestricted use, distribution, and reproduction in any medium, provided the original work is properly cited.

A pebbling move on a graph $G$ consists of taking two pebbles off one vertex and placing one pebble on an adjacent vertex. The pebbling number of a connected graph $G$, denoted by $f(G)$, is the least $n$ such that any distribution of $n$ pebbles on $G$ allows one pebble to be moved to any specified but arbitrary vertex by a sequence of pebbling moves. This paper determines the pebbling numbers and the 2-pebbling property of the middle graph of fan graphs.

\section{Introduction}

Pebbling on graphs was first introduced by Chung [1]. Consider a connected graph with a fixed number of pebbles distributed on its vertices. A pebbling move consists of the removal of two pebbles from a vertex and the placement of one of those pebbles on an adjacent vertex. The pebbling number of a vertex $v$ in a graph $G$ is the smallest number $f(G, v)$ with the property that from every placement of $f(G, v)$ pebbles on $G$, it is possible to move a pebble to $v$ by a sequence of pebbling moves. The pebbling number of a graph $G$, denoted by $f(G)$, is the maximum of $f(G, v)$ over all the vertices of $G$.

In a graph $G$, if each vertex (except $v$ ) has at most one pebble, then no pebble can be moved to $v$. Also, if $u$ is of distance $d$ from $v$ and at most $2^{d}-1$ pebbles are placed on $u$ (and none elsewhere), then no pebble can be moved from $u$ to $v$. So it is clear that $f(G) \geq \max \left\{|V(G)|, 2^{D}\right\}$, where $|V(G)|$ is the number of vertices of $G$ and $D$ is the diameter of $G$.

Throughout this paper, let $G$ be a simple connected graph with vertex set $V(G)$ and edge set $E(G)$. For a distribution of pebbles on $G$, denote by $p(H)$ and $p(v)$ the number of pebbles on a subgraph $H$ of $G$ and the number of pebbles on a vertex $v$ of $G$, respectively. In addition, denote by $\widetilde{p}(H)$ and $\tilde{p}(v)$ the number of pebbles on $H$ and the number of pebbles on $v$ after a specified sequence of pebbling moves, respectively. For $u v \in$ $E(G), u \stackrel{m}{\rightarrow} v$ refers to taking $2 m$ pebbles off $u$ and placing $m$ pebbles on $v$. Denote by $\left\langle v_{1}, v_{2}, \ldots, v_{n}\right\rangle$ the path with vertices $v_{1}, v_{2}, \ldots, v_{n}$ in order.
We now introduce some definitions and give some lemmas, which will be used in subsequent proofs.

Definition 1. A fan graph, denoted by $F_{n}$, is a path $P_{n-1}$ plus an extra vertex $v_{0}$ connected to all vertices of the path $P_{n-1}$, where $P_{n-1}=\left\langle v_{1}, v_{2}, \ldots, v_{n-1}\right\rangle$.

Definition 2. The middle graph $M(G)$ of a graph $G$ is the graph obtained from $G$ by inserting a new vertex into every edge of $G$ and by joining by edges those pairs of these new vertices which lie on adjacent edges of $G$.

Now one creates the middle graph of $F_{n}$. Edges $v_{1} v_{2}, v_{2} v_{3}, \ldots, v_{(n-2)(n-1)}$ of $F_{n}$ are the inserted new vertices $u_{12}, u_{23}, \ldots, u_{(n-2)(n-1)}$ in the sequence, and edges $v_{0} v_{1}, v_{0} v_{2}, \ldots, v_{0} v_{n-1}$ of $F_{n}$ are the inserted new vertices $u_{01}, u_{02}, \ldots, u_{0(n-1)}$, respectively. By joining by edges those pairs of these inserted vertices which lie on adjacent edges of $F_{n}$, this obtains the middle graph of $F_{n}$ (see Figure 1).

Definition 3. A transmitting subgraph is a path $\left\langle v_{0}, v_{1}, \ldots, v_{k}\right\rangle$ such that there are at least two pebbles on $v_{0}$, and after a sequence of pebbling moves, one can transmit a pebble from $v_{0}$ to $v_{k}$.

Lemma 4 (see [2]). Let $P_{k+1}=\left\langle v_{0}, v_{1}, \ldots, v_{k}\right\rangle$. If

$$
p\left(v_{0}\right)+2 p\left(v_{1}\right)+\cdots+2^{i} p\left(v_{i}\right)+\cdots+2^{k-1} p\left(v_{k-1}\right) \geq 2^{k},
$$

then $P_{k+1}$ is a transmitting subgraph. 


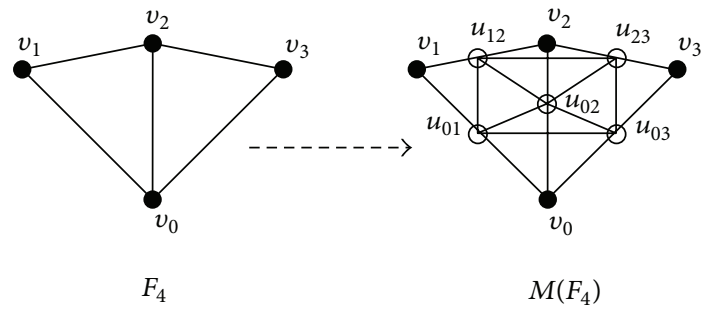

Figure 1: $M\left(F_{4}\right)$.

Definition 5. The $t$-pebbling number, $f_{t}(G)$, of a connected graph, $G$, is the smallest positive integer such that from every placement of $f_{t}(G)$ pebbles, $t$ pebbles can be moved to a specified target vertex by a sequence of pebbling moves.

Lemma 6 (see [3]). If $K_{n}$ is the complete graph with $n(n \geq 2)$ vertices, then $f_{t}\left(K_{n}\right)=2 t+n-2$.

Lemma 7 (see [4]). Consider $f\left(M\left(P_{n}\right)\right)=2^{n}+n-2$.

Chung found the pebbling numbers of the $n$-cube $Q^{n}$, the complete graph $K_{n}$, and the path $P_{n}$ (see [1]). The pebbling number of $C_{n}$ was determined in [5]. In $[6,7]$, Ye et al. gave the number of squares of cycles. Feng and Kim proved that $f\left(F_{n}\right)=n$ and $f\left(W_{n}\right)=n$ (see [8]). Liu et al. determined the pebbling numbers of middle graphs of $P_{n}, K_{n}$, and $K_{1, n-1}$ (see [4]). In [9], Ye et al. proved that $f\left(M\left(C_{2 n}\right)\right)=2^{n+1}+2 n-$ $2(n \geq 2)$ and $f\left(M\left(C_{2 n+1}\right)\right)=\left\lfloor 2^{n+3} / 3\right\rfloor+2 n$, where $M\left(C_{n}\right)$ denotes the middle graph of $C_{n}$. Motivated by these works, we will determine the value of the pebbling number and the 2-property of middle graphs of $F_{n}$.

\section{Pebbling Numbers of $M\left(F_{n}\right)$}

In this section, we study the pebbling number of $M\left(F_{n}\right)$. Let $S=\left\{v_{0}, u_{01}, u_{02}, \ldots, u_{0(n-1)}\right\}$, and let $A=\left\{v_{1}, u_{12}, v_{2}, u_{23}, \ldots, v_{n-1}\right\}$. Obviously, the subgraph induced by $S$ is a complete graph with $n$ vertices. For $n=3$, $M\left(F_{3}\right) \cong M\left(C_{3}\right)$. Hence we have the following theorem.

Theorem 8 (see [9]). Consider $f\left(M\left(F_{3}\right)\right)=7$.

Lemma 9. Let $f\left(M\left(F_{n-1}\right)\right)=p$. If $p+3$ pebbles are placed on $M\left(F_{n}\right)$, then one pebble can be moved to any specified vertex of $S$ by a sequence of pebbling moves.

Proof. Let $v$ be our target vertex, and let $p(v)=0$, where $v \in S$. We may assume that $v \neq u_{01}$ (after relabeling if necessary). Let $B=\left\{v_{1}, u_{12}, u_{01}\right\}$. If $p(B) \geq 5$, then $\widetilde{p}\left(u_{01}\right) \geq 2$ by Lemma 6 , and we can move one pebble to $v$. If $p(B)=4$, then $B \stackrel{1}{\rightarrow} u_{02}$. We delete $v_{1}, u_{01}$, and $u_{12}$ to obtain the subgraph $M\left(F_{n-1}\right)$ with $p$ pebbles, thus we can move one pebble to $v$. If $p(B) \leq 3$, then we delete $v_{1}, u_{01}$, and $u_{12}$ to obtain the subgraph $M\left(F_{n-1}\right)$ with at least $p$ pebbles and we are done.

Theorem 10. Consider $f\left(M\left(F_{4}\right)\right)=11$.
Proof. We place 7 pebbles on $v_{3}$ and one pebble on each vertex of the set $\left\{v_{0}, u_{02}, v_{2}\right\}$, other vertices have no pebble, then no pebble can be moved to $v_{1}$. So $p\left(M\left(F_{4}\right)\right) \geq 11$. We now place 11 pebbles on $M\left(F_{4}\right)$. We assume that $v$ is our target vertex and $p(v)=0$. Recall $S=\left\{v_{0}, u_{01}, u_{02}, u_{03}\right\}$ and $A=$ $\left\{v_{1}, u_{12}, v_{2}, u_{23}, v_{3}\right\}$

(1) Consider $v \in S$. By Theorem 8 and Lemma 9, we can move one pebble to $v$.

(2) Consider $v=v_{1}$ (or $\left.v=v_{3}\right)$. Let $A_{1}=A-\left\{v_{1}\right\}$, let $A_{2}=\left\{u_{12}, v_{2}\right\}$, and let $A_{3}=A_{1}-A_{2}$. If $p(S)=t$, then $p\left(A_{1}\right)=11-t$. Thus we can move at least $\lfloor(8-t) / 2\rfloor$ pebbles from $A_{1}$ to $S$ so that $\tilde{p}(S)=\lfloor(8+t) / 2\rfloor \geq 6$ for $t \geq 4$. By Lemma $6, \widetilde{p}\left(u_{01}\right)=2$ and we can move one pebble to $v_{1}$. If $t \leq 2$, then $p(A) \geq 9$. By Lemma 7 , we can move one pebble to $v_{1}$. If $t=3$, then at least one of $u_{01}$ and $u_{03}$ can obtain one pebble from every placement of 3 pebbles on $S$ by a sequence of pebbling moves. If $p\left(A_{3}\right) \geq 7$, then $A_{3} \stackrel{3}{\rightarrow} u_{03}$. So $\left\langle u_{03}, u_{01}, v_{1}\right\rangle$ is a transmitting subgraph. If $4 \leq p\left(A_{3}\right) \leq 6$, then $2 \leq$ $p\left(A_{2}\right) \leq 4$. By Lemma $6, \widetilde{p}\left(u_{23}\right) \geq 2$ and $\widetilde{p}\left(u_{12}\right) \geq 1$. So $\left\langle u_{23}, u_{12}, v_{1}\right\rangle$ is a transmitting subgraph. If $p\left(A_{3}\right) \leq$ 3 , then $p\left(A_{2}\right) \geq 5$. So $\left\langle v_{2}, u_{12}, v_{1}\right\rangle$ is a transmitting subgraph.

(3) Consider $v=v_{2}$. If $p(S) \geq 4$ or $p(S) \leq 2$, then we are done with (2). If $p(S)=3$, then $p\left(v_{1}\right)+p\left(u_{12}\right) \geq 4$ or $p\left(u_{23}\right)+p\left(v_{3}\right) \geq 4$. So $\left\langle v_{1}, u_{12}, v_{2}\right\rangle$ or $\left\langle v_{3}, u_{23}, v_{2}\right\rangle$ is a transmitting subgraph.

(4) Consider $v=u_{12}$ (or $v=u_{23}$ ). If $p(S) \geq 4$ or $p(S) \leq 2$, then we are done with (2). If $p(S)=3$, then $p\left(v_{1}\right)+p\left(v_{2}\right)+p\left(u_{23}\right)+p\left(v_{3}\right)=8$. Obviously, we are done if $p\left(v_{1}\right) \geq 2$ or $p\left(v_{2}\right) \geq 2$. Next suppose that $p\left(v_{1}\right) \leq 1$ and $p\left(v_{2}\right) \leq 1$. Thus $p\left(u_{23}\right)+p\left(v_{3}\right) \geq 6$. So $\left\langle v_{3}, u_{23}, u_{12}\right\rangle$ is a transmitting subgraph.

Theorem 11. Consider $f\left(M\left(F_{n}\right)\right)=3 n-1(n \geq 4)$.

Proof. We place 7 pebbles on $v_{n-1}$ and one pebble on each vertex of $M\left(F_{n}\right)$ except $v_{1}, u_{01}, u_{12}, u_{(n-2)(n-1)}, u_{0(n-1)}$, and $v_{n-1}$. In this configuration of pebbles, we cannot move one pebble to $v_{1}$. So $f\left(M\left(F_{n}\right)\right) \geq 3 n-1$. Next, let us use induction on $n$ to show that $f\left(M\left(F_{n}\right)\right)=3 n-1$. For $n=4$, our theorem is true by Theorem 10. Suppose that $f\left(M\left(F_{k}\right)\right)=3 k-1$ if $k<n$. Now $3 n-1$ pebbles are placed arbitrarily on the vertices of $M\left(F_{n}\right)$. Suppose that $v$ is our target vertex and $p(v)=0$.

(1) Consider $v \in S$. By induction and Theorem 8 , we can move one pebble to $v$.

(2) Consider $v=v_{1}$ (or $\left.v=v_{n-1}\right)$. Obviously, $p\left(u_{01}\right) \leq 1$. Otherwise, $p\left(u_{01}\right)>1$. $v_{1}$ can obtain one pebble. Let $B_{i}=$ $\left\{u_{i(i+1)}, u_{0(i+1)}, v_{i+1}\right\}(1 \leq i \leq n-2)$.

If $p\left(B_{n-2}\right) \leq 3$, then we delete $B_{n-2}$ to obtain the subgraph $M\left(F_{n-1}\right)$ with at least $3(n-1)-1$ pebbles. By induction, we can move one pebble to $v_{1}$. If $p\left(B_{n-2}\right)=4$, then $B_{n-2} \stackrel{1}{\rightarrow} u_{0(n-2)}$. Thus we delete $B_{n-2}$ to obtain the subgraph $M\left(F_{n-1}\right)$ with $3(n-1)-1$ pebbles. By induction, we are done. 
Next, suppose that $p\left(B_{n-2}\right) \geq 5$. By Lemma $6, \widetilde{p}\left(u_{0(n-1)}\right) \geq$ 2. If $p\left(u_{01}\right)=1$, then $\left\langle u_{0(n-1)}, u_{01}, v_{1}\right\rangle$ is a transmitting subgraph. If $p\left(v_{0}\right) \geq 2$, then $v_{0} \stackrel{1}{\rightarrow} u_{01}$, and we are done. If there exists some $B_{i}$ with $p\left(B_{i}\right) \geq 5(i \neq n-2)$, then $B_{i} \stackrel{1}{\rightarrow} u_{01}$, and we are done. Thus we assume that $p\left(u_{01}\right)=0, p\left(v_{0}\right) \leq 1$, and $p\left(B_{i}\right) \leq 4$ for $1 \leq i \leq n-3$.

Now, we consider $B_{i}(1 \leq i \leq n-3)$. Clearly, if $p\left(B_{1}\right)=$ 4 , then we are done. Suppose that there exists some $B_{j}$ with $p\left(B_{j}\right)=4(j \neq 1)$. It is clear that if one of the three cases $((i)$ $p\left(u_{0 j}\right) \geq 1\left(u_{0 j} \in B_{j-1}\right)$, (ii) $p\left(B_{j-1}\right) \geq 3$, and (iii) $p\left(v_{j}\right) \geq 2$ $\left.\left(v_{j} \in B_{j-1}\right)\right)$ happens, then we can move one pebble to $v$. Thus we assume that $p\left(B_{i}\right)=4(2 \leq i \leq n-3), p\left(B_{i-1}\right) \leq 2, p\left(u_{0 i}\right)=$ 0 , and $p\left(v_{i}\right) \leq 1$. If there are $r$ sets $B_{i_{1}}, B_{i_{2}}, \ldots, B_{i_{r}}$ such that $p\left(B_{i_{k}}\right)=4$ for $1 \leq k \leq r$, then $p\left(B_{i_{k}-1}\right) \leq 2$ for $1 \leq k \leq r$. Let $N_{1}=\left\{i_{1}, i_{2}, \ldots, i_{r}\right\}$, let $N_{2}=\left\{i_{1}-1, i_{2}-1, \ldots, i_{r}-1\right\}$, and let $N_{3}=\{1,2, \ldots, n-3\}-N_{1}-N_{2}$. If $p\left(B_{j}\right)=2$ for all $j \in N_{2}$ and $p\left(B_{k}\right)=3$ for all $k \in N_{3}$, then $\widetilde{p}\left(u_{j(j+1)}\right)=1$ and $\widetilde{p}\left(u_{k(k+1)}\right)=1$. Recall that $p\left(B_{i}\right)=4$ for all $i \in N_{1}$ and $p\left(B_{n-2}\right) \geq 5$. Then $\widetilde{p}\left(u_{i(i+1)}\right)=1$ and $\widetilde{p}\left(u_{(n-2)(n-1)}\right)=2$. Thus $\left\langle u_{(n-2)(n-1)}, u_{(n-3)(n-2)}, \ldots, u_{12}, v_{1}\right\rangle$ is a transmitting subgraph. So there is at least some $j$ in $N_{2}$ such that $p\left(B_{j}\right) \leq 1$ or at least some $k$ in $N_{3}$ such that $p\left(B_{k}\right) \leq 2$. If there are two $j^{\prime}$ and $j^{\prime \prime}$ in $N_{2}$ such that $p\left(B_{j^{\prime}}\right) \leq 1$ and $p\left(B_{j^{\prime \prime}}\right) \leq 1$ or two $k^{\prime}$ and $k^{\prime \prime}$ in $N_{3}$ such that $p\left(B_{k^{\prime}}\right) \leq 2$ and $p\left(B_{k^{\prime \prime}}\right) \leq 2$ or some $j$ in $N_{2}$ such that $p\left(B_{j}\right) \leq 1$ and some $k$ in $N_{3}$ such that $p\left(B_{k}\right) \leq 2$, then $p\left(B_{n-2}\right) \geq 9$. By Lemma $6, \widetilde{p}\left(u_{0(n-1)}\right)=4$. Hence $\left\langle u_{0(n-1)}, u_{01}, v_{1}\right\rangle$ is a transmitting subgraph.

Finally, there are two remaining cases, (i) there is only some $j$ in $N_{2}$ such that $p\left(B_{j}\right) \leq 1$, and (ii) there is only some $k$ in $N_{3}$ such that $p\left(B_{k}\right) \leq 2$. So $p\left(B_{n-2}\right) \geq 8$. If $p\left(u_{(n-2)(n-1)}\right)=0$, then $\left\langle v_{n-1}, u_{0(n-1)}, u_{01}, v_{1}\right\rangle$ is a transmitting subgraph. If $p\left(u_{(n-2)(n-1)}\right) \neq 0$, then, in $B_{n-2}, \widetilde{p}\left(u_{(n-2)(n-1)}\right) \geq$ 2 and $\widetilde{p}\left(u_{0(n-1)}\right) \geq 2$. For (i), we have $\widetilde{p}\left(u_{i(i+1)}\right) \geq 1$ for $j+2 \leq i \leq n-3$. By the transmitting subgraph $\left\langle u_{(n-2)(n-1)}, u_{(n-3)(n-2)}, \ldots, u_{(j+1)(j+2)}\right\rangle, \widetilde{p}\left(B_{j+1}\right)=5$ and we are done. Suppose that (ii) holds. If $p\left(B_{k}\right)=2$, then we can move one pebble from $u_{0(n-1)}$ to $u_{0(k+1)}$ so that $p\left(B_{k}\right)=3$, and we are done. If $p\left(B_{k}\right) \leq 1$, then $p\left(B_{n-2}\right) \geq 9$ and we are done.

(3) Consider $v=u_{12}$ (or $\left.v=u_{(n-2)(n-1)}\right)$. Obviously, $p\left(u_{01}\right) \leq 1$ and $p\left(v_{i}\right) \leq 1(i=1,2)$. Otherwise, one pebble can be moved to $u_{12}$. The proof is similar to (2).

(4) Consider $v=v_{i}(2 \leq i \leq n-2)$ (or $v=u_{j(j+1)}(2 \leq$ $j \leq n-3))$ and $p\left(v_{i}\right)=0$. Let $B=\left\{v_{1}, u_{12}, u_{01}\right\}$, and let $B^{\prime}=\left\{v_{n-1}, u_{(n-2)(n-1)}, u_{0(n-1)}\right\}$. If $p(B) \leq 3$, then delete $B$ to obtain the subgraph $M\left(F_{n-1}\right)$ with at least $3(n-1)-1$ pebbles. By induction, we can move one pebble to $v$. If $p(B)=4$, then we can move one pebble from $B$ to $u_{02}$, after deleting $B$ to obtain the subgraph $M\left(F_{n-1}\right)$ with $3(n-1)-1$ pebbles. Hence we assume that $p(B) \geq 5$. According to symmetry, $p\left(B^{\prime}\right) \geq 5$. Therefore we are done.

\section{The 2-Pebbling Property of $M\left(F_{n}\right)$}

For a distribution of pebbles on $G$, let $q$ be the number of vertices with at least one pebble. We say a graph $G$ satisfies the 2-pebbling property if two pebbles can be moved to any specified vertex when the total starting number of pebbles is $2 f(G)-q+1$. Next we will discuss the 2-pebbling property of $M\left(F_{n}\right)$. For the convenience of statement, let $S=$ $\left\{x_{1}, x_{2}, \ldots, x_{n}\right\}$, and let $A=\left\{y_{1}, y_{2}, \ldots, y_{2 n-3}\right\}$, where $x_{1}=v_{0}$, $x_{2}=u_{01}, \ldots, x_{n}=u_{0(n-1)}, y_{1}=v_{1}$, and $y_{2}=u_{12}, \ldots, y_{2 n-3}=$ $v_{n-1}$. In this section let $q=q_{s}+q_{a}$, where $q_{s}$ and $q_{a}$ are the number of vertices with at least one pebble in $S$ and $A$, respectively.

Lemma 12. Suppose that $p\left(M\left(F_{n}\right)\right) \geq 2(3 n-1)-q$ and $q_{a}=$ $2 n-4$. If $p(S)=q_{s}+t(t=0,1,2)$ and $p\left(y_{r}\right)=0(1 \leq r \leq$ $2 n-3)$, then one can move 2 pebbles to $y_{r}$.

Proof. Let $r=2 k-1$ (or $r=2 k$ ). Since $q_{a}=2 n-4$ and $p(S)=q_{s}+t$, so $p(A) \geq 4 n+2-2 q_{s}-t$. Without loss of generality, there exists a positive integer $j(j>r)$ such that the corresponding vertex $y_{j}$ with $p\left(y_{j}\right) \geq 2$ and $p\left(y_{i}\right)=1$ for $r+1 \leq i \leq j-1$. Thus $y_{j} \stackrel{1}{\rightarrow} y_{j-1} \stackrel{1}{\rightarrow} \cdots \stackrel{1}{\rightarrow} y_{r}$. Using the remaining $4 n+2-t-2 q_{s}-(j-r+1)$ pebbles on vertices $y_{1}, y_{2}, \ldots, y_{r-1}, y_{j}, y_{j+1}, \ldots, y_{2 n-3}$, we can move at least $n+$ $\lfloor(5-t) / 2\rfloor-q_{s}$ pebbles to $S$ so that $\widetilde{p}(S) \geq n+\lfloor(5+t) / 2\rfloor$. By Lemma $6, \tilde{p}\left(x_{k+1}\right)=2$. So we can move one additional pebble from $x_{k+1}$ to $y_{r}$ so that $\tilde{p}\left(y_{r}\right)=2$.

Lemma 13. Suppose that $p\left(M\left(F_{n}\right)\right)=2(3 n-1)-q+1$ and $q_{a}=2 n-5$. If $p(S)=q_{s}+t(t=0,1)$ and $p\left(y_{r}\right)=0(1 \leq r \leq$ $2 n-3)$, then one can move 2 pebbles to $y_{r}$.

Proof. Let $r=2 k-1$ (or $r=2 k$ ). Since $q_{a}=2 n-5$, we see that there is only some vertex $y_{i_{0}}\left(i_{0} \neq r\right)$ with $p\left(y_{i_{0}}\right)=0$. Without loss of generality, there exists a positive integer $j(j>r)$ such that the corresponding vertex $y_{j}$ with $p\left(y_{j}\right) \geq 2$ and $p\left(y_{i}\right) \leq 1$ for $r<i<j$. If $i_{0}=2 k_{0}-1\left(k_{0} \neq k\right)$ or $i_{0} \notin\{r+1, r+2, \ldots, j-1\}$, then we can move one pebble to $y_{r}$ by the transmitting subgraph $\left\langle y_{j}, y_{j-2}, \ldots, y_{r+1}, y_{r}\right\rangle$ or $\left\langle y_{j}, y_{j-1}, y_{j-3}, \ldots, y_{r+1}, y_{r}\right\rangle$. Now using the remaining at least $4 n+4-t-2 q_{s}-(j-r+1)$ pebbles on the set $A_{1}=$ $\left\{y_{1}, y_{2}, \ldots, y_{r-1}, y_{j}, y_{j}, \ldots, y_{2 n-3}\right\}$, we can move $n+\lfloor(7-$ $t) / 2\rfloor-q_{s}$ pebbles from the $A_{1}$ to $S$ so that $\tilde{p}(S)=n+\lfloor(7+t) / 2\rfloor$. By Lemma 6, $\widetilde{p}\left(x_{k+1}\right)=2$ and we can move one additional pebble from $x_{k+1}$ to $y_{r}$ so that $\tilde{p}\left(y_{r}\right)=2$.

Suppose that $i_{0}=2 k_{0}\left(k_{0} \geq k\right)$ and $i_{0} \in\{r+1, r+2, \ldots, j-$ $1\}$. If $j=i_{0}+1$, then $y_{j} \stackrel{1}{\rightarrow} y_{i_{0}}$. Thus there must exist one vertex $y_{j^{\prime}}\left(j^{\prime} \geq j\right)$ with $p\left(y_{j^{\prime}}\right) \geq 2$ and $p\left(y_{i}\right) \leq 1$ for $r<i<$ $j^{\prime}$. Using the transmitting subgraph $\left\langle y_{j^{\prime}}, y_{j^{\prime}-2}, \ldots, y_{r+1}, y_{r}\right\rangle$ or $\left\langle y_{j^{\prime}}, y_{j^{\prime}-1}, y_{j^{\prime}-3}, \ldots, y_{r+1}, y_{r}\right\rangle$, we can move one pebble to $y_{r}$. Now, using the remaining $4 n+4-t-2 q_{s}-\left(j^{\prime}-r+\right.$ 2) pebbles on the set $\left\{y_{1}, y_{2}, \ldots, y_{r-1}, y_{j^{\prime}}, y_{j^{\prime}+1}, \ldots, y_{2 n-3}\right\}$, we can move $n+\lfloor(6-t) / 2\rfloor-q_{s}$ pebbles from the set $\left\{y_{1}, y_{2}, \ldots, y_{r-1}, y_{j^{\prime}}, y_{j^{\prime}+1}, \ldots, y_{2 n-3}\right\}$ to $S$ so that $\widetilde{p}(S) \geq n+$ $\lfloor(6+t) / 2\rfloor$. By Lemma $6, \widetilde{p}\left(x_{k+1}\right)=2$ and we are done. Next, suppose that $j \geq i_{0}+2$.

(1) Consider $p\left(y_{2 k}\right)=1$. We divide into three subcases.

(1.1) Consider $p\left(x_{k+2}\right)=0$. We delete vertices $y_{r}, y_{r+1}, \ldots, y_{2 k_{0}}, x_{k+2}$ to obtain the subgraph with two sets $A_{2}=A-\left\{y_{r}, y_{r+1}, \ldots, y_{2 k_{0}}\right\}$ and $S_{1}=S-\left\{x_{k+2}\right\}$, and $p\left(A_{2}\right)=4 n+4-2 q_{s}-t-\left(2 k_{0}-r-1\right)$ and $p\left(S_{1}\right)=q_{s}+t$. Thus we can move $n+\lfloor(10-t) / 2\rfloor-q_{s}$ pebbles from $A_{2}$ to 
$S_{1}$ so that $\widetilde{p}\left(S_{1}\right)=n+\lfloor(10+t) / 2\rfloor$. By Lemma $6, \widetilde{p}\left(x_{k+1}\right)=4$ and we can move two pebbles from $x_{k+1}$ to $y_{r}$.

(1.2) Consider $p\left(x_{k+2}\right)=1$. Suppose that $j=2 k^{\prime}$ or $j=$ $2 k^{\prime}+1\left(k^{\prime}>k\right)$. Let $A_{3}=\left\{y_{2 k^{\prime}}, y_{2 k^{\prime}+1}\right\}$. Obviously, $p\left(A_{3}\right) \geq 3$. If $p\left(A_{3}\right) \geq 5$, then

$$
A_{3} \stackrel{2}{\longrightarrow} x_{k^{\prime}+2} \stackrel{1}{\longrightarrow} x_{k+2} \stackrel{1}{\longrightarrow} y_{r+1} \stackrel{1}{\longrightarrow} y_{r} .
$$

We delete $y_{r}, y_{r+1}, \ldots, y_{2 k_{0}}, x_{k+2}$ to obtain the subgraph with two sets $A_{2}$ and $S_{1}$. So $p\left(A_{2}\right)=4 n-2 q_{s}-t-\left(2 k_{0}-r-1\right)$ and $\tilde{p}\left(S_{1}\right)=q_{s}-1+t$. We can move $n+\lfloor(6-t) / 2\rfloor-q_{s}$ pebbles from $A_{2}$ to $S_{1}$ so that $\tilde{p}\left(S_{1}\right)=n+\lfloor(4+t) / 2\rfloor$. By Lemma 6, $\widetilde{p}\left(x_{k+1}\right)=2$ and we are done. If $p\left(A_{3}\right)=3,4$ and $p\left(x_{k^{\prime}+2}\right) \neq 0$, then

$$
A_{3} \stackrel{1}{\longrightarrow} x_{k^{\prime}+2} \stackrel{1}{\longrightarrow} x_{k+2} \stackrel{1}{\longrightarrow} y_{r+1} \stackrel{1}{\longrightarrow} y_{r} .
$$

We delete $y_{r}, y_{r+1}, \ldots, y_{2 k_{0}}, x_{k+2}$ to obtain the subgraph with two sets $A_{2}$ and $S_{1}$. So $p\left(A_{2}\right)=4 n+2-2 q_{s}-t-\left(2 k_{0}-r-1\right)$ and $\widetilde{p}\left(S_{1}\right)=q_{s}-2+t$. We can move $n+\lfloor(8-t) / 2\rfloor-q_{s}$ pebbles from $A_{2}$ to $S_{1}$ so that $\widetilde{p}\left(S_{1}\right)=n+\lfloor(4+t) / 2\rfloor$. By Lemma $6, \widetilde{p}\left(x_{k+1}\right)=$ 2 and we are done. If $p\left(A_{3}\right)=3,4$ and $p\left(x_{k^{\prime}+2}\right)=0$, then $A_{3} \stackrel{1}{\rightarrow} x_{k^{\prime}+1}$. We delete $y_{r}, y_{r+1}, \ldots, y_{2 k_{0}}, y_{2 k^{\prime}}, y_{2 k^{\prime}+1}, x_{k^{\prime}+2}$ to obtain the subgraph with two sets $A_{4}=A_{2}-A_{3}$ and $S_{2}=$ $S-\left\{x_{2 k^{\prime}+2}\right\}$. So $p\left(A_{4}\right) \geq 4 n-2 q_{s}-t-\left(2 k_{0}-r-1\right)$ and $\widetilde{p}\left(S_{2}\right)=q_{s}+1+t$. We can move $n+\lfloor(8-t) / 2\rfloor-q_{s}$ pebbles from $A_{4}$ to $S_{2}$ so that $\widetilde{p}\left(S_{2}\right)=n+\lfloor(10+t) / 2\rfloor$. By Lemma 6 , $\widetilde{p}\left(x_{k+1}\right)=4$.

(1.3) Consider $p\left(x_{k+2}\right)=2$ for $t=1$. Thus $x_{k+2} \stackrel{1}{\rightarrow} y_{2 k} \stackrel{1}{\rightarrow}$ $y_{r}$. We delete $y_{r}, y_{r+1}, \ldots, y_{2 k_{0}}, x_{k+2}$ to obtain the subgraph with two sets $A_{2}$ and $S_{1}$. So $p\left(A_{2}\right)=4 n+3-2 q_{s}-\left(2 k_{0}-r-1\right)$ and $\tilde{p}\left(S_{1}\right)=q_{s}-1 . n+4-q_{s}$ pebbles can be moved from $A_{2}$ to $S_{1}$ so that $\widetilde{p}\left(S_{1}\right)=n+3$. By Lemma $6, \widetilde{p}\left(x_{k+1}\right)=3$. So we can move one additional pebble from $x_{k+1}$ to $y_{r}$.

(2) Consider $p\left(y_{2 k}\right)=0$; that is, $k=k_{0}$. We divide into three subcases.

(2.1) Consider $p\left(x_{2 k+2}\right)=0$. We delete $y_{r}, y_{r+1}, y_{r+2}, x_{2 k+2}$ to obtain the subgraph with two sets $A_{5}=A-\left\{y_{r}, y_{r+1}, y_{r+2}\right\}$ and $S_{1}$. One has $p\left(A_{5}\right)=4 n+3-2 q_{s}-t$ and $p\left(S_{1}\right)=q_{s}+t$. We can move $n+\lfloor(10-t) / 2\rfloor-q_{s}$ pebbles from $A_{5}$ to $S_{1}$ so that $\widetilde{p}\left(S_{1}\right)=n+\lfloor(10+t) / 2\rfloor$. By Lemma $6, \widetilde{p}\left(x_{k+1}\right)=4$ and we can move two pebbles from $x_{k+1}$ to $y_{r}$.

(2.2) Consider $p\left(x_{k+2}\right)=1$. We have

$$
y_{j} \stackrel{1}{\longrightarrow} y_{j-1} \stackrel{1}{\longrightarrow} \cdots \stackrel{1}{\longrightarrow} y_{r+2} \stackrel{1}{\longrightarrow} x_{k+2} \stackrel{1}{\longrightarrow} x_{k+1} .
$$

We delete vertices $y_{r}, y_{r+1}, \ldots, y_{j-1}, x_{k+2}$ to obtain the subgraph with two sets $A_{1}$ and $S_{1}$. So $p\left(A_{1}\right)=4 n+4-2 q_{s}-t-$ $(j-r)$ and $\widetilde{p}\left(S_{1}\right)=q_{s}+t-1$ (except one moved pebble on $\left.x_{k+1}\right)$. We can move $n+\lfloor(8-t) / 2\rfloor-q_{s}$ pebbles from $A_{5}$ to $S_{1}$ so that $\widetilde{p}\left(S_{1}\right)=n+\lfloor(6+t) / 2\rfloor$ (except one moved pebble on $\left.x_{k+1}\right)$. By Lemma 6 , we can move 3 additional pebbles to $x_{k+1}$ so that $\widetilde{p}\left(x_{k+1}\right)=4$.

(2.3) $p\left(x_{k+2}\right)=2$ for $t=1$. Thus $x_{k+2} \stackrel{1}{\rightarrow} x_{k+1}$. Deleting $y_{r}, y_{r+1}, y_{r+2}, x_{k+2}$ to obtain the subgraph with two sets $A_{5}$ and $S_{1}$. One has $p\left(A_{5}\right)=4 n+2-2 q_{s}$ and $\widetilde{p}\left(S_{1}\right)=q_{s}-1$ (except one moved pebble on $\left.x_{k+1}\right)$. We can move $n+4-q_{s}$ pebbles from $A_{4}$ to $S_{1}$ so that $\widetilde{p}\left(S_{1}\right)=n+3$ (except one moved pebble on $\left.x_{k+1}\right)$. By Lemma 6, we can move 3 additional pebbles to $x_{k+1}$ so that $\tilde{p}\left(x_{k+1}\right)=4$.

Theorem 14. $M\left(F_{n}\right)$ has the 2-pebbling property.

Proof. Suppose that $v$ is our target vertex. If $p(v)=1$, then the number of pebbles on $M\left(F_{n}\right)$ except one pebble on $v$ is $2(3 n-1)+1-q-1(>3 n-1)$. By Theorem 11 , we can move one additional pebble to $v$ so that $\widetilde{p}(v)=2$. Next we assume that $p(v)=0$.

(1) Consider $v=x_{r}(1 \leq r \leq n)$. If there exists some vertex $x_{i}$ with $p\left(x_{i}\right) \geq 2(i \neq r)$, then $x_{i} \stackrel{1}{\rightarrow} x_{r}$. Using the remaining $2(3 n-1)+1-q-2>3 n-1$ pebbles, we can move one additional pebble to $x_{r}$ so that $\widetilde{p}\left(x_{r}\right)=2$. If $p\left(x_{i}\right) \leq 1$ for $1 \leq i \leq n$, then $p(A)=2(3 n-1)-q+1-q_{s}=6 n-1-q_{a}-2 q_{s} \geq 4 n+2-2 q_{s}$. Thus we can move at least $n+2-q_{s}$ pebbles from $A$ to $S$ so that $\widetilde{p}(S)=n+2$. By Lemma 6 , we can move two pebbles to $x_{r}$.

(2) Consider $v=y_{r}(1 \leq r \leq 2 n-3)$. Let $r=2 k-1$ (or $r=2 k$ ). If $p\left(x_{k+1}\right) \geq 2$, then we can put one pebble on $y_{r}$. After that, the remaining $2(3 n-1)-q+1-2(>3 n-1)$ pebbles on $M\left(F_{n}\right)$ suffice to put one additional pebble on $y_{r}$ by Theorem 11. Next we assume $p\left(x_{k+1}\right) \leq 1$.

(2.1) Suppose that $p\left(x_{k+1}\right)=1$. If there is some vertex $x_{i}$ with $p\left(x_{i}\right) \geq 2(i \neq k+1)$, then $x_{i} \stackrel{1}{\rightarrow} x_{k+1} \stackrel{1}{\rightarrow} y_{r}$. The remaining $2(3 n-1)-q+1-3(>3 n-1)$ pebbles on $M\left(F_{n}\right)$ will suffice to put one additional pebble on $y_{r}$ so that $\widetilde{p}\left(y_{r}\right)=2$. Next we assume that $p\left(x_{i}\right) \leq 1$ for $1 \leq i \leq n$. Obviously, $p(S)=q_{s}$ and $p(A)=2(3 n-1)-q+1-q_{s}=6 n-1-q_{a}-2 q_{s}$. If $q_{a} \leq 2 n-5$, then $p(A) \geq 4 n+4-2 q_{s}$. Thus we can move at least $n+5-q_{s}$ pebbles from $A$ to $S$ so that $\tilde{p}(S)=n+5$. By Lemma 6 , we can move 3 additional pebbles to $x_{k+1}$ so that $\widetilde{p}\left(x_{k+1}\right)=4$ and we are done. If $q_{a}=2 n-4$, then, by Lemma 12 , we are done.

(2.2) Suppose that $p\left(x_{k+1}\right)=0$. If we can find some vertex $x_{i}$ with $p\left(x_{i}\right) \geq 4$ or find two vertices $x_{j}$ with $p\left(v_{j}\right) \geq 2$ and $x_{j^{\prime}}$ with $p\left(x_{j^{\prime}}\right) \geq 2$, then using 4 pebbles on $x_{i}$ or two pebbles on $x_{j}$ and two pebbles on $x_{j^{\prime}}$ we can move one pebble to $y_{r}$. Then the remaining $2(3 n-1)-q+1-4(>3 n-1)$ pebbles on $M\left(F_{n}\right)$ will suffice to put one additional pebble to $y_{r}$ so that $\widetilde{p}\left(y_{r}\right)=2$.

Consider only some vertex $x_{i}$ with $2 \leq p\left(x_{i}\right) \leq 3$. If $p\left(x_{i}\right)=3$, then $x_{i} \stackrel{1}{\rightarrow} x_{k+1}, \widetilde{p}(S)=q_{s}$, and $p(A)=2(3 n-1)-$ $q_{s}-q_{a}+1-\left(q_{s}+2\right)=6 n-3-2 q_{s}-q_{a}$. When $q_{a} \leq 2 n-5$ and $p(A) \geq 4 n+2-2 q_{s}$, we can move at least $n+4-q_{s}$ pebbles from $A$ to $S$ so that $\widetilde{p}(S) \geq n+4$ except for one pebble on $x_{k+1}$. By Lemma 6, we can put 3 additional pebbles on $x_{k+1}$ so that $\widetilde{p}\left(x_{k+1}\right)=4$. When $q_{a}=2 n-4$, we are done with Lemma 12. If $p\left(x_{i}\right)=2$, then $x_{i} \stackrel{1}{\rightarrow} x_{k+1}, \tilde{p}(S)=q_{s}-1$, and $p(A)=2(3 n-1)-q_{s}-q_{a}+1-\left(q_{s}+1\right)=6 n-2-2 q_{s}-q_{a}$. When $q_{a} \leq 2 n-6$ and $p(A) \geq 4 n+4-2 q_{s}$, we can move at least $n+5-q_{s}$ pebbles from $A$ to $S$ so that $\widetilde{p}(S) \geq n+4$ except for one pebble on $x_{k+1}$. By Lemma 6 , we can put 3 additional pebbles on $x_{k+1}$ so that $\tilde{p}\left(x_{k+1}\right)=4$. When $q_{a}=2 n-4$ and $q_{a}=2 n-5$, we are done with Lemmas 12 and 13 .

Consider $p\left(x_{i}\right) \leq 1$ for $1 \leq i \leq n$. Obviously, $p(S)=$ $q_{s}$ and $p(A)=6 n-1-q_{a}-2 q_{s}$. When $q_{a} \leq 2 n-6$ 
and $p(A) \geq 4 n+5-2 q_{s}$, we can move at least $n+6-q_{s}$ pebbles from $A$ to $S$ so that $\widetilde{p}(S) \geq n+6$. By Lemma $6, \widetilde{p}\left(x_{k+1}\right)=4$ and we are done. When $q_{a}=2 n-4$ and $q_{a}=2 n-5$, we are done with Lemmas 12 and 13.

\section{Conflict of Interests}

The authors declare that there is no conflict of interests regarding the publication of this paper.

\section{Acknowledgments}

This work is supported by National Natural Science Foundation of China (no. 10971248) and Anhui Provincial Natural Science Foundation (nos. 1408085MA08 and KJ2013Z279).

\section{References}

[1] F. R. K. Chung, "Pebbling in hypercubes," SIAM Journal on Discrete Mathematics, vol. 2, no. 4, pp. 467-472, 1989.

[2] H. S. Snevily and J. A. Foster, "The 2-pebbling property and a conjecture of Graham's," Graphs and Combinatorics, vol. 16, no. 2, pp. 231-244, 2000.

[3] A. Lourdusamy and S. Somasundaram, "The $t$-pebbling number of graphs," Southeast Asian Bulletin of Mathematics, vol. 30, no. 5, pp. 907-914, 2006.

[4] H. Y. Liu, Q. Qin, Z. P. Wang, and Y. G. Ma, "Pebbling number, of middle graphs," Journal of Dalian Maritime University, vol. 32, no. 4, pp. 125-128, 2006.

[5] L. Pachter, H. S. Snevily, and B. Voxman, "On pebbling graphs," Congressus Numerantium, vol. 107, pp. 65-80, 1995.

[6] Y. S. Ye, M. Q. Zhai, and Y. Zhang, "Pebbling number of squares of odd cycles," Discrete Mathematics, vol. 312, no. 21, pp. 31743178, 2012.

[7] Y. Ye, P. Zhang, and Y. Zhang, "The pebbling number of squares of even cycles," Discrete Mathematics, vol. 312, no. 21, pp. 32033211, 2012.

[8] R. Feng and J. Y. Kim, "Pebbling numbers of some graphs," Science in China A, vol. 45, no. 4, pp. 470-478, 2002.

[9] Y. S. Ye, F. Liu, and M. Q. Zhai, "Pebbling numbers of middle graphs of cycles and Graham's conjecture," Operations Research Transactions, vol. 17, no. 3, pp. 35-44, 2013. 


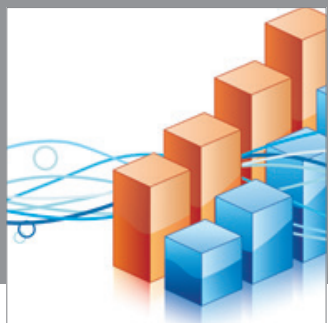

Advances in

Operations Research

mansans

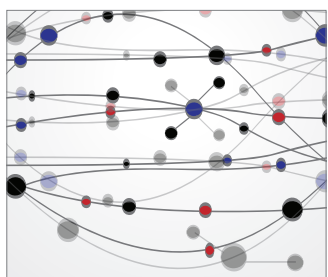

The Scientific World Journal
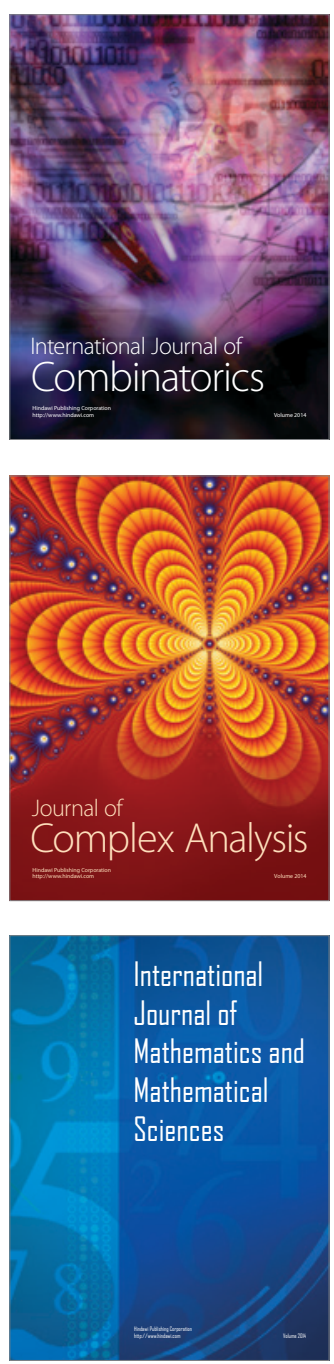
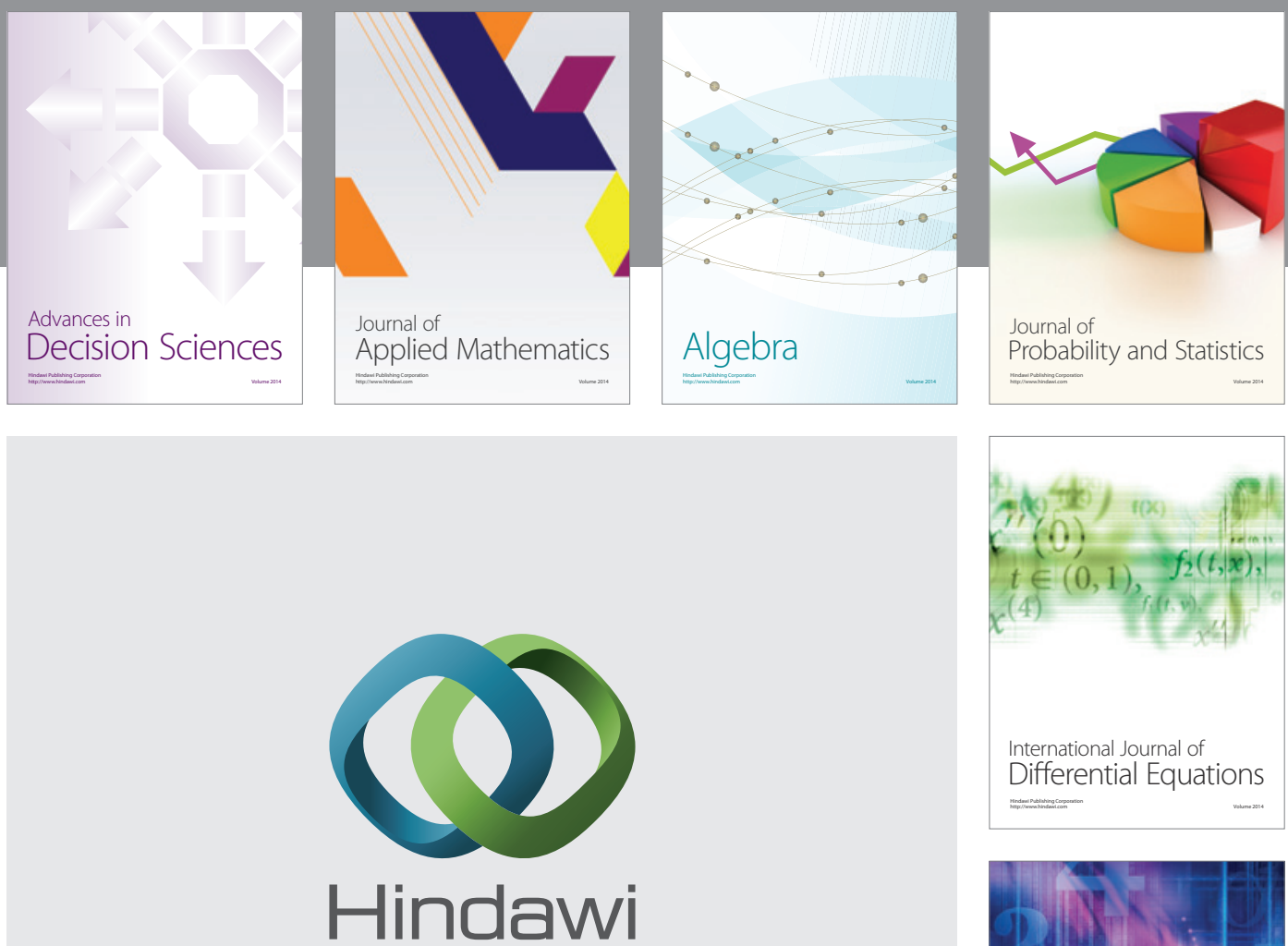

Submit your manuscripts at http://www.hindawi.com
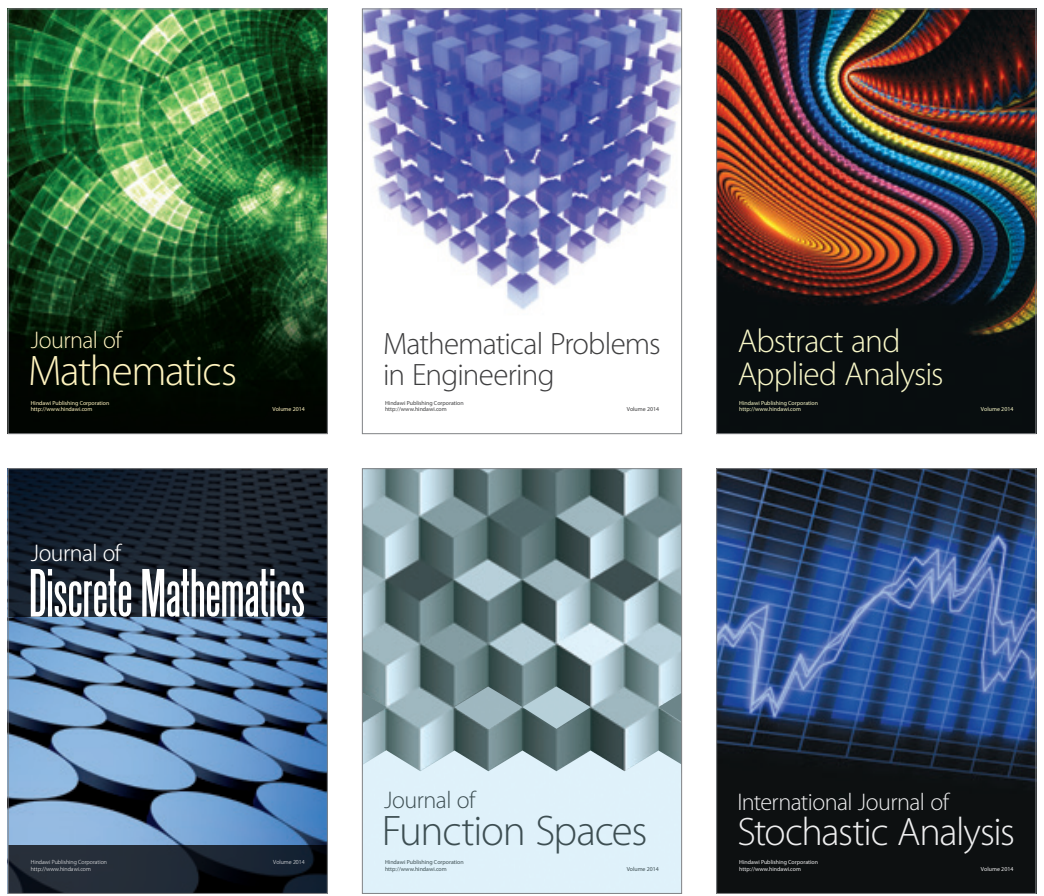

Journal of

Function Spaces

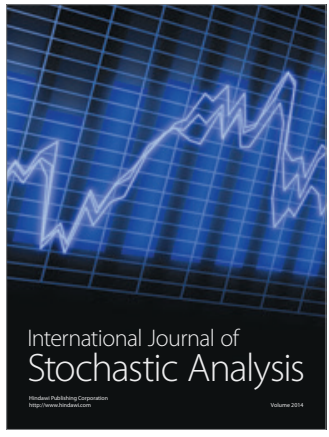

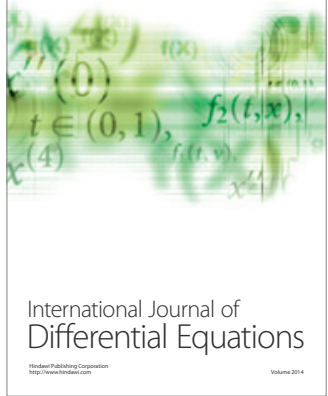
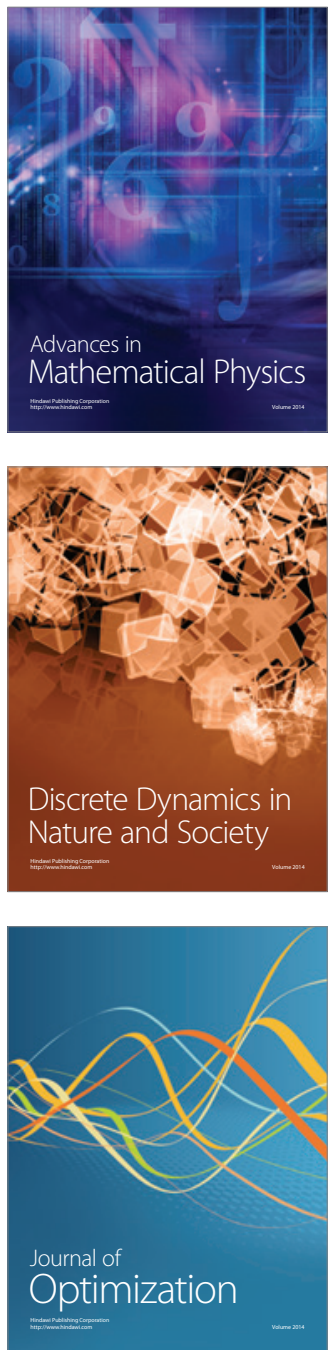